\title{
PROBABILISTIC CHARACTERIZATION OF CERTAIN BANACH SPACES
}

\author{
Dedicated to Professor Hisaharu Umegaki on his 60th birthday
}

\author{
By JUN KAWABE
}

\section{Introduction.}

Let $(\Omega, \mathcal{A}, P)$ be a probability measure space, $X$ a real separable Banach space and $X^{*}$ its topological dual space. In this paper we look into the relation between the convergence of a sequence $\left(\xi_{n}\right)_{n \geqq 1}$ of random variables with values in $X$ (for short, random elements) and the convergence of the associated sequence $\left(\left\langle\xi_{n}, f\right\rangle\right)_{n \geqq 1}, f \in X^{*}$, of real random variables. In [8], when $X$ is a Hilbert space, the author has treated this problem for mean square convergence with the aid of covariance operators or more specifically, with the aid of the relative compactness of covariance operators with respect to the trace norm. In ChevetChobanjan-Linde-Tarieladze $[1,10]$ they showed that covariance operators on Banach spaces introduced in Vakhania [14] are nuclear operators which were defined by Grothendieck [6]. This leads us to consider the following

Proposition (A) $\xi_{n}$ converges in $L_{2}(\Omega ; X)$ if and only if the following two conditions are satisfied: line.

(a) For each $f \in X^{*},\left\langle\xi_{n}, f\right\rangle$ converges in $L_{2}(\Omega ; \boldsymbol{R})$, where $\boldsymbol{R}$ is the real

(b) The set $\left\{R_{\xi_{n}}\right\}$ of covariance operators is relatively compact with respect to the nuclear norm.

This Proposition (A), however, is not valid in general. The purpose of this paper is to find necessary and sufficient conditions on the structure of Banach spaces in order that the Proposition (A) holds.

In section 2 we shall give some definitions and preliminary results. In Section 3 we shall show that the Proposition (A) is not valid unless $X$ is isomorphic to a Hilbert space. This result will be proved by an isomorphic characterization of Hilbert spaces which was obtained by Kwapień [9]. In Section 4 we shall also prove that the Proposition (A) holds only for Gaussian random elements if and only if $X$ is of type 2 .

The author thanks Professor Hisaharu Umegaki for his valuable advice and encouragement. He also thanks Professor Fumio Hiai who carefully read the

Received March 19, 1985 
text of this paper and gave him some useful comments.

\section{Preliminaries.}

Let $X$ be a real separable Banach space, $X^{*}$ its topological dual space and $\mathscr{B}(X)$ the Borel field of $X$. By a random element in $X$ defined on a basic probability space $(\Omega, \mathcal{A}, P)$ we mean a measurable mapping $\xi:(\Omega, \mathcal{A}) \rightarrow(X, \mathscr{B}(X))$. Every random element $\xi$ induces on $(X, \mathcal{B}(X))$ the probability measure $\mu_{\xi}=P_{\circ} \xi^{-1}$ which is called its distribution. Let $\mu$ be a probability measure on $X$. The characteristic functional of $\mu$ is defined by

$$
\hat{\mu}(f)=\int_{X} \exp \{\imath\langle x, f\rangle\} \mu(d x), \quad f \in X^{*} .
$$

We say that a sequence $\left(\mu_{n}\right)_{n \geqq 1}$ of probability measures on $X$ converges weakly if there exists a probability measure $\mu$ on $X$ such that

$$
\int_{X} \varphi(x) \mu_{n}(d x) \rightarrow \int_{X} \varphi(x) \mu(d x) \quad \text { as } n \rightarrow \infty
$$

for every bounded continuous real function $\varphi$ on $X$. It is well known ([12]) that the topology on probability measures determined by the above weak convergence is metrizable by Prokhorov metric. A set $\left\{\xi_{\alpha}\right\}$ of random elements in $X$ is said to be uniformly tight if for each $\varepsilon>0$, there exists a compact subset $C_{\varepsilon}$ of $X$ such that

$$
\mu_{\xi_{\alpha}}\left(C_{\varepsilon}\right)>1-\varepsilon \quad \text { for all } \alpha .
$$

Let us recall that a subset $A$ of a metric space is said to be relatively compact if every sequence in $A$ contains a convergent subsequence. According to [12] the set $\left\{\xi_{\alpha}\right\}$ is uniformly tight if and only if the set $\left\{\mu_{\xi_{\alpha}}\right\}$ is relatively compact with respect to Prokhorov metric.

Denote by $L_{2}(\Omega ; X)$ the Banach space of all random elements in $X$ which satisfy

$$
E\|\xi\|^{2} \equiv \int_{\Omega}\|\xi(\omega)\|^{2} P(d \omega)<\infty,
$$

where $E$ denotes the expectation with respect to $P$. We shall also denote by $M(\Omega ; X)$ the Fréchet space of all random elements in $X$ with the metric $\rho$ given by

$$
\rho(\xi, \eta)=E\left\{\frac{\|\xi-\eta\|}{1+\|\xi-\eta\|}\right\}, \quad \xi, \eta \in M(\Omega ; X) .
$$

We say that a sequence $\left(\xi_{n}\right)_{n \geq 1}$ converges in probability if there exists a $\xi \in M(\Omega ; X)$ such that $\rho\left(\xi_{n}, \xi\right) \rightarrow 0$ as $n \rightarrow \infty$. It is well known that $\xi_{n}$ converges in probability to $\xi$ if and only if, for each $\varepsilon>0, P\left(\left\|\xi_{n}-\xi\right\| \geqq \varepsilon\right) \rightarrow 0$ as $n \rightarrow \infty$. Most of the relations which exist among the various modes of convergence for real random variables are also valid for random elements. For instance, almost sure 
convergence or convergence in $L_{2}(\Omega ; X)$ imply convergence in probability, and convergence in probability implies weak convergence, and hence uniform tightness. When $X$ is the real line we abbreviate $L_{2}(\Omega ; X)$ and $M(\Omega ; X)$ to $L_{2}(\Omega)$ and $M(\Omega)$ respectively. A random element $\xi$ in $X$ is said to be Gaussian if for each $f \in X^{*},\langle\xi, f\rangle$ is a (possibly degenerate) real Gaussian random variable on $(\Omega$, $\mathcal{A}, P)$. Every Gaussian random element belongs to $L_{2}(\Omega ; X)$ (see Fernique [5]).

Let $X, Y$ be Banach spaces. A linear operator $T: X \rightarrow Y$ is said to be nuclear if it admits the representation

$$
T x=\sum_{k=1}^{\infty}\left\langle x, f_{k}\right\rangle y_{k}, \quad x \in X,
$$

where $f_{k} \in X^{*}, \quad y_{k} \in Y$ and $\sum_{k=1}^{\infty}\left\|f_{k}\right\|\left\|y_{k}\right\|<\infty$. The greatest lower bound of the sum $\sum_{k=1}^{\infty}\left\|f_{k}\right\|\left\|y_{k}\right\|<\infty$ taken over all possible representations of the operator $T$ is called the nuclear norm of $T$ and denoted by $\nu(T)$ (see [6]). We shall denote by $\Re(X, Y)$ the Banach space of all nuclear operators from $X$ into $Y$ with the nuclear norm $\nu(\cdot)$. A linear operator $R: X^{*} \rightarrow X$ is said to be positive if $\langle R f, f\rangle \geqq 0$ for all $f \in X^{*}$ and symmetric if $\langle R f, g\rangle=\langle R g, f\rangle$ for all $f, g \in X^{*}$. According to $[1,10]$, for every $\xi \in L_{2}(\Omega ; X)$ there exist a mean vector $m_{\xi} \in X$ and a covariance operator $R_{\xi} \in \Re\left(X^{*}, X\right)$ such that

and

$$
\left\langle m_{\xi}, f\right\rangle=E\langle\xi, f\rangle, \quad f \in X^{*}
$$

$$
\left\langle R_{\xi} f, g\right\rangle=E\left\langle\xi-m_{\xi}, f\right\rangle\left\langle\xi-m_{\xi}, g\right\rangle, \quad f, g \in X^{*} .
$$

Then $R_{\xi}$ is positive and symmetric, and has the estimate

$$
\nu\left(R_{\xi}\right) \leqq E\left\|\xi-m_{\xi}\right\|^{2} .
$$

Let $\left(\gamma_{n}\right)_{n \geq 1}$ be a sequence of independent standard Gaussian random variables. A Banach space $X$ is said to be of type 2 if for each sequence $\left(x_{n}\right)_{n \geqq 1}$ in $X$ with the property $\sum_{n=1}^{\infty}\left\|x_{n}\right\|^{2}<\infty$, the series $\sum_{n=1}^{\infty} \gamma_{n} x_{n}$ is convergent a.s. (=almost surely) and is said to be of cotype 2 if for each sequence $\left(x_{n}\right)_{n \geq 1}$ satisfying that the series $\sum_{n=1}^{\infty} \gamma_{n} x_{n}$ is convergent a.s., we have always $\sum_{n=1}^{\infty}\left\|x_{n}\right\|^{2}<\infty$. For instance, the spaces $l_{p}, L_{p}(2 \leqq p<\infty)$ are of type 2 and they are of cotype 2 when $1 \leqq p \leqq 2$. We know that if $X$ is of type 2 and of cotype 2 then it is isomorphic to a Hilbert space (see [9]).

\section{Characterization of Hilbert spaces.}

We now characterize Hilbert spaces by mean square convergence of random elements.

THEOREM 1. For a real separable Banach space $X$, the following assertions (1)-(3) are equivalent:

(1) $X$ is isomorphic to a Hilbert space. 
(2) For any subset $K$ of mean-zero random elements in $L_{2}(\Omega ; X), K$ is relatively compact if (and only if) the following two conditions are satısfied

(a) For each $f \in X^{*}$, the set $\{\langle\xi, f\rangle ; \xi \in K\}$ is relatively compact in $L_{2}(\Omega)$.

(b) The set $\left\{R_{\xi} ; \xi \in K\right\}$ is relatively compact in $\Re\left(X^{*}, X\right)$.

(3) For any sequence $\left(\xi_{n}\right)_{n \geqq 1}$ of mean-zero random elements in $L_{2}(\Omega ; X), \xi_{n}$ converges if (and only if) the following two conditions are satisfied

(c) For each $f \in X^{*},\left\langle\xi_{n}, f\right\rangle$ converges in $L_{2}(\Omega)$.

(d) The set $\left\{R_{\xi_{n}}\right\}$ is relatively compact in $\Re\left(X^{*}, X\right)$.

We need the following two results to prove Theorem 1 .

THEOREM $\mathcal{K}$ ([8; Proposition 1$])$. Let $H$ be a real separable Hilbert space with inner product $(\cdot, \cdot)$. A set $K \subset L_{2}(\Omega ; H)$ is relatively compact if and only if the following two conditions are satisfied:

(a) For each $x \in H$, the set $\{(\xi, x) ; \xi \in K\}$ is relatively compact in $L_{2}(\Omega)$.

(b) The set $\left\{m_{\xi} ; \xi \in K\right\}$ is relatively compact in $H$ and the set $\left\{R_{\xi} ; \xi \in K\right\}$ is relatively compact in $\Re(H, H)$ (i.e. the space of all trace class operators on $H$ ).

The following lemma follows immediately from the definition of the nuclear norm and we state it without proof.

Lemma 1. Let $X$ be a real separable Banach space and $\xi \in L_{2}(\Omega ; X)$. Then we have for every $f \in X^{*}$,

$$
E\left|\left\langle\xi-m_{\xi}, f\right\rangle\right|^{2} \leqq\|f\|^{2} \cdot \nu\left(R_{\xi}\right) .
$$

Proof of Theorem 1. The implication $(1) \Rightarrow(2)$ follows from Theorem $\mathcal{K}$. $(2) \Rightarrow(3)$ : It sufficies to show that $\xi_{n}$ converges in $L_{2}(\Omega ; X)$ if we assume conditions (c) and (d). Since $\left\{\xi_{n}\right\}$ satisfies the conditions (a) and (b) of the assumption (2) we see that the set $\left\{\xi_{n}\right\}$ is relatively compact in $L_{2}(\Omega ; X)$. Now we shall assume that $\xi_{n}$ is not convergent. From this and the relative compactness of the set $\left\{\xi_{n}\right\}$ we can find two subsequences $\left(\xi_{n^{\prime}}\right)$ and $\left(\xi_{n^{*}}\right)$ converging in $L_{2}(\Omega ; X)$ to distinct elements $\xi_{1}$ and $\xi_{2}$ respectively. Then from (c) it follows that for each $f \in X^{*}$ we have $\left\langle\xi_{1}(\omega), f\right\rangle=\left\langle\xi_{2}(\omega), f\right\rangle$ a.s. On the other hand, since $X$ is separable, by the Hahn-Banach theorem we can find a countable subset $\left\{g_{k}\right\}$ of $X^{*}$ which separates points in $X$. Then the standard argument shows that there exists a $P$-null set $N$ such that, if $\omega \notin N,\left\langle\xi_{1}(\omega), g_{k}\right\rangle=\left\langle\xi_{2}(\omega), g_{k}\right\rangle$ for all $k \geqq 1$. Thus $\xi_{1}=\xi_{2}$ a. s. and this is a contradiction.

$(3) \Rightarrow(1)$ : From the result of [9] we shall prove that $X$ is of type 2 and of cotype 2. First we prove that $X$ is of type 2 . Let $\left(x_{\imath}\right)_{i \geq 1}$ be a sequence in $X$ such that

$$
\sum_{i=1}^{\infty}\left\|x_{i}\right\|^{2}<\infty
$$

and we define mean-zero Gaussian random elements as follows: 


$$
\xi_{n}(\cdot)=\sum_{i=1}^{n} \gamma_{i}(\cdot) x_{i} \quad n=1,2, \cdots,
$$

where $\left(\gamma_{2}\right)_{i \geqq 1}$ is a sequence of independent standard Gaussian random variables. Then by a routine calculation using the fact that

we have

$$
E\left[\gamma_{i} \gamma_{3}\right]=\left\{\begin{array}{lll}
1 & \text { if } & \imath=\jmath \\
0 & \text { if } & i \neq j
\end{array}\right.
$$

$$
R_{\xi_{n}} f=\sum_{\imath=1}^{n}\left\langle x_{\imath}, f\right\rangle x_{\imath}, \quad f \in X^{*} .
$$

Then $\left(\xi_{n}\right)_{n \geq 1}$ satisfies the conditions (c) and (d) of the assumption (3). To see this it is sufficient to show that $\left(\left\langle\xi_{n}, f\right\rangle\right)_{n \geqq 1}$ is a Cauchy sequence in $L_{2}(\Omega)$ for every $f \in X^{*}$ and $\left(R_{\xi_{n}}\right)_{n \geqq 1}$ is also a Cauchy sequence in $\Re\left(X^{*}, X\right)$. By (3.3) we have for each $f \in X^{*}$ and all integers $n>m \geqq 1$,

$$
\begin{aligned}
E\left|\left\langle\xi_{n}, f\right\rangle-\left\langle\xi_{m}, f\right\rangle\right|^{2} & =E\left|\sum_{\imath=m+1}^{n}\left\langle x_{\imath}, f\right\rangle \gamma_{\imath}\right|^{2} \\
& =\sum_{\imath, j=m+1}^{n}\left\langle x_{\imath}, f\right\rangle\left\langle x_{\jmath}, f\right\rangle E\left[\gamma_{\imath} \gamma_{\jmath}\right] \\
& =\sum_{\imath=m+1}^{n}\left\langle x_{\imath}, f\right\rangle^{2} \leqq\|f\|^{2} \sum_{i=m+1}^{n}\left\|x_{i}\right\|^{2} .
\end{aligned}
$$

On the other hand, by (3.4) we have for all $n>m \geqq 1$

$$
\left(R_{\xi_{n}}-R_{\xi_{m}}\right) f=\sum_{\imath=m+1}^{n}\left\langle x_{\imath}, f\right\rangle x_{\imath} .
$$

Therefore from the definition of the nuclear norm we get

$$
\nu\left(R_{\xi_{n}}-R_{\xi_{m}}\right) \leqq \sum_{\imath=m+1}^{n}\left\|x_{i}\right\|^{2}
$$

Thus by (3.1), (3.5) and (3.6) we see that $\left(\left\langle\xi_{n}, f\right\rangle\right)_{n \geqq 1}$ and $\left(R_{\xi_{n}}\right)_{n \geqq 1}$ are Cauchy sequences in $L_{2}(\Omega)$ and in $\Re\left(X^{*}, X\right)$ respectively. Therefore $\left(\xi_{n}\right)_{n \geqq 1}$ satisfies conditions (c) and (d), so that $\xi_{n}=\sum_{\imath=1}^{n} \gamma_{\imath} x_{\imath}$ converges in $L_{2}(\Omega ; X)$ by the assumption (3). Consequently, using Itô-Nisio's theorem [7], $\sum_{\imath=1}^{\infty} \gamma_{\imath} x_{\imath}$ is convergent a. s., that is, $X$ is of type 2 .

Next we prove that $X$ is of cotype 2. Assume that there exists a sequence $\left(x_{\imath}\right)_{i \geq 1}$ such that $\sum_{\imath=1}^{\infty} \gamma_{\imath} x_{\imath}$ is convergent a. s., but $\sum_{\imath=1}^{\infty}\left\|x_{i}\right\|^{2}=\infty$. If we set $a_{k}=\sum_{l=1}^{k}\left\|x_{i}\right\|^{2}$ then $a_{k} \rightarrow \infty$ and also $\sum_{k=1}^{\infty}\left\|x_{k}\right\|^{2} / a_{k}=\infty$. Define a sequence $\left(\zeta_{k}\right)_{k \geqq 1}$ of independent mean-zero random elements in $L_{2}(\Omega ; X)$ with distributions such that

$$
P\left(\zeta_{k}=-\frac{a_{k}^{1 / 2} x_{k}}{\left\|x_{k}\right\|}\right)=P\left(\zeta_{k}=\frac{a_{k}^{1 / 2} x_{k}}{\left\|x_{k}\right\|}\right)=\frac{1}{2} \cdot \frac{\left\|x_{k}\right\|^{2}}{a_{k}}
$$




$$
P\left(\zeta_{k}=0\right)=1-\frac{\left\|x_{k}\right\|^{2}}{a_{k}} .
$$

Then, by the Borel-Cantelli lemma, $\zeta_{k}$ does not converge to 0 a. s. so that

$$
\eta_{n} \equiv \sum_{k=1}^{n} \zeta_{k} \quad \text { diverges a.s. }
$$

Let us consider the sequence $\left(\xi_{n}\right)_{n \geq 1}$ of mean-zero Gaussian random elements defined by (3.2). Then, since $\xi_{n}=\sum_{\imath=1}^{n} \gamma_{\imath} x_{\imath}$ is convergent a. s., by [3; Theorem 1] $R_{\xi_{n}}$ converges in $\mathscr{N}\left(X^{*}, X\right)$, and hence the set $\left\{R_{\xi_{n}}\right\}$ is relatively compact in $\Re\left(X^{*}, X\right)$. On the other hand, from (3.4) and Lemma 1 we have for all $n \geqq 1$

so that

$$
\begin{aligned}
\sum_{\imath=1}^{n}\left\langle x_{\imath}, f\right\rangle^{2} & =E\left|\left\langle\xi_{n}, f\right\rangle\right|^{2} \\
& \leqq\|f\|^{2} \cdot \nu\left(R_{\xi_{n}}\right) \leqq\|f\|^{2} \cdot \sup _{n} \nu\left(R_{\xi_{n}}\right)<\infty,
\end{aligned}
$$

$$
\sum_{\imath=1}^{\infty}\left\langle x_{\imath}, f\right\rangle^{2} \quad \text { is convergent. }
$$

Now we show that $\left(\eta_{n}\right)_{n \geq 1}$ defined by (3.7) satisfies the conditions (c) and (d) of the assumption (3). To see this it sufficies to show that $\left(\left\langle\eta_{n}, f\right\rangle\right)_{n \geqq 1}$ is a Cauchy sequence in $L_{2}(\Omega)$ for every $f \in X^{*}$ and the set $\left\{R_{\eta_{n}}\right\}$ is relatively compact in $\Re\left(X^{*}, X\right)$. A routine calculation using the fact that

$$
E\left\langle\zeta_{\imath}, f\right\rangle\left\langle\zeta_{\jmath}, g\right\rangle=\left\{\begin{array}{cc}
\left\langle x_{\imath}, f\right\rangle\left\langle x_{\jmath}, g\right\rangle & \text { if } \imath=j \\
0 & \text { if } \imath \neq j
\end{array}\right.
$$

shows that for each $f \in X^{*}$ and all integers $n>m \geqq 1$,

$$
\begin{aligned}
E\left|\left\langle\eta_{n}, f\right\rangle-\left\langle\eta_{m}, f\right\rangle\right|^{2} & =E\left|\sum_{k=m+1}^{n}\left\langle\zeta_{k}, f\right\rangle\right|^{2} \\
& =\sum_{k=m+1}^{n}\left\langle x_{k}, f\right\rangle^{2} .
\end{aligned}
$$

We see from (3.8) that $\left(\left\langle\eta_{n}, f\right\rangle\right)_{n \geqq 1}$ is a Cauchy sequence in $L_{2}(\Omega)$. On the other hand, by (3.4) and (3.9) it is easy to show that $R_{\xi_{n}}=R_{\eta_{n}}$ for all $n \geqq 1$. From this and the relative compactness of the set $\left\{R_{\xi_{n}}\right\}$, the set $\left\{R_{\eta_{n}}\right\}$ must be relatively compact in $\Re\left(X^{*}, X\right)$. Thus from the assumption (3) it follows that $\eta_{n}=\sum_{k=1}^{n} \zeta_{k}$ is convergent in $L_{2}(\Omega ; X)$. Consequently, by Itô-Nisio's theorem [7], $\sum_{k=1}^{\infty} \zeta_{k}$ is convergent a. s. and this contradicts (3.7). The proof is now complete.

\section{Characterization of Banach spaces of type 2.}

We now turn to characterization of Banach spaces of type 2 by the convergence in probability of (mean-zero) Gaussian random elements. Let $X$ be 
a real separable Banach space. A subset $G$ of $X^{*}$ is said to be total if $G$ separates points in $X$, that is, $\langle x, g\rangle=0$ for all $g \in G$ implies $x=0$. Before formulating Theorem 2 below we shall prove the following results of interest in themselves.

LEMMA 2. Let $\left(\xi_{n}\right)_{n \geqq 1}$ be a sequence in $M(\Omega ; X)$. Then $\xi_{n}$ converges in probability if and only if the following two conditions are satisfied

(a) For each $g \in G,\left\langle\xi_{n}, g\right\rangle$ converges in probability, where $G$ is a total subset of $X^{*}$.

(b) The set $\left\{\xi_{n}\right\}$ is uniformly tight.

Proof. The proof of the necessity is obvious. We proceed by a variation of an argument in $[7 ;$ p. 38] in order to prove the sufficiency. Let us denote the distributions of $\xi_{n}-\xi_{m}$ by $\mu_{m n}, m, n \geqq 1$. Then from (b) it is easy to show that the set $\left\{\mu_{m n} ; m, n \geqq 1\right\}$ is uniformly tight. We shall now prove that $\xi_{n}$ converges in probability. Suppose to the contrary that there exist $\varepsilon>0$ and a subsequence $\left(\mu_{m^{\prime} n^{\prime}}\right)$ such that

$$
\mu_{m^{\prime} n^{\prime}}\left(B_{\varepsilon}\right) \leqq 1-\varepsilon \quad \text { for all } m^{\prime}, n^{\prime},
$$

where $B_{\varepsilon}$ denotes the open $\varepsilon$-neighborhood of the origin in $X$. Since $\left\{\mu_{m n}\right\}$ is uniformly tight, we can assume that $\mu_{m^{\prime} n^{\prime}}$ converges weakly to a probability measure $\mu$ on $X$, so that

$$
\mu\left(B_{\varepsilon}\right) \leqq \liminf _{\substack{m^{\prime} \rightarrow \infty \\ n^{\prime} \rightarrow \infty}} \mu_{m^{\prime} n^{\prime}}\left(B_{\varepsilon}\right) \leqq 1-\varepsilon .
$$

On the other hand we have

$$
\left.\widehat{\mu_{m^{\prime} n^{\prime}}}(f)=E\left[\exp \left(i<\xi_{n^{\prime}}-\xi_{m^{\prime}}, f\right\rangle\right)\right], \quad f \in X^{*} .
$$

If we denote by $S(G)$ the linear subspace spanned by $G$ in $X^{*}$ then it is easy from (a) to show that $\left\langle\xi_{n^{\prime}}-\xi_{m^{\prime}}, f\right\rangle$ converges to 0 in probability for every $f \in S(G)$. Consequently, letting $m^{\prime}, n^{\prime} \rightarrow \infty$ in (4.2), we have $\hat{\mu}(f)=1$ for every $f \in S(G)$, so that $\mu$ concentrates at the origin in $X$ by Perlman [11; Theorem 9]. This contradicts (4.1) and the proof is now complete.

COROllary 1. A set $K \subset M(\Omega ; X)$ is relatively compact if and only if the following two conditions are satisfied:

(a) For each $g \in G$, the set $\{\langle\xi, g\rangle ; \xi \in K\}$ is relatively compact in $M(\Omega)$, where $G$ is a total subset of $X^{*}$.

(b) $K$ is uniformly tight.

Proof. The proof of the necessity is obvious. To prove the sufficiency, let $\left(\xi_{n}\right)_{n \geq 1}$ be an arbitrary sequence in $K$, and show that $\left(\xi_{n}\right)_{n \geq 1}$ contains a subsequence converging in probability. Since $X$ is separable, by the Hahn-Banach theorem we can get a countable total subset $G$ of $X^{*}$. By (a), using a diagonal selection argument, we can find a subsequence $\left(\xi_{n^{\prime}}\right)$ such that for each $g \in G$, 
$\left\langle\xi_{n^{\prime}}, g\right\rangle$ converges in probability. On the other hand, it is obvious from (b) that the set $\left\{\xi_{n^{\prime}}\right\}$ is uniformly tight. Thus $\xi_{n^{\prime}}$ converges in probability since $\left(\xi_{n^{\prime}}\right)$ satisfies the conditions (a) and (b) of Lemma 2. The proof is now complete.

We say that a Banach space $X$ has the approximation property if, for every compact set $C$ in $X$ and every $\varepsilon>0$, there is a continuous linear operator $T$ : $X \rightarrow X$ of finite rank such that $\|T x-x\| \leqq \varepsilon$ for every $x \in C$. Though, by Enflo's example, not all Banach spaces enjoy this property, many spaces, for instance, $l_{p}, L_{p}(1 \leqq p \leqq \infty)$ and $C[0,1]$, have enough structure to allow this approximation by operators of finite rank. (See [6] and Diestel-Uhl [4].)

Let $\xi$ and $\xi_{n}, n=1,2, \cdots$, be mean-zero Gaussian random elements. It is known in [3] that $\lim _{n \rightarrow \infty} \nu\left(R_{\xi_{n}}-R_{\xi}\right)=0$ if $\mu_{\xi_{n}}$ converges weakly to $\mu_{\xi}$, but the converse implication is not valid in general. However, we have the following theorem which was given by Chevet.

THEOREM $\mathcal{C}$ ([2]). For a real separable Banach space $X$ which has the approximation property, the following assertions (1)-(3) are equivalent:

(1) $X$ is of type 2.

(2) For any set $K$ of mean-zero Gaussian random elements in $X, K$ is uniformly tight if (and only if) the set $\left\{R_{\xi} ; \xi \in K\right\}$ is relatively compact in $\Re\left(X^{*}, X\right)$.

(3) For any sequence $\left(\xi_{n}\right)_{n \geq 0}$ of mean-zero Gaussian random elements in $X$, $\mu_{\xi_{n}}$ converges weakly to $\mu_{\xi_{0}}$ if (and only if) $\lim _{n \rightarrow \infty} \nu\left(R_{\xi_{n}}-R_{\xi_{0}}\right)=0$.

For the case of convergence in probability we have the following:

THEOREM 2. For a real separable Banach space $X$ which has the approximation property, the following assertions (1)-(3) are equivalent:

(1) $X$ is of type 2.

(2) For any set $K$ of mean-zero Gaussian random elements in $X, K$ is relatively compact in $M(\Omega ; X)$ if (and only if) the following two conditions are satisfied:

(a) For each $f \in X^{*}$, the set $\{\langle\xi, f\rangle$; $\xi \in K\}$ is relatively compact in $M(\Omega)$.

(b) The set $\left\{R_{\xi} ; \xi \in K\right\}$ is relatively compact in $\Re\left(X^{*}, X\right)$.

(3) For any sequence $\left(\xi_{n}\right)_{n \geqq 1}$ of mean-zero Gaussian random elements in $X$, $\xi_{n}$ converges in probability if (and only if) the following two conditions are satisfied:

(c) For each $f \in X^{*},\left\langle\xi_{n}, f\right\rangle$ converges in probability.

(d) The set $\left\{R_{\xi_{n}}\right\}$ is relatively compact in $\Re\left(X^{*}, X\right)$.

Proof. The implication $(1) \Rightarrow(2)$ follows from Theorem $\mathcal{C}$ and Corollary 1 . $(2) \Rightarrow(3)$ : We proceed in a way similar to the proof of the implication $(2) \Rightarrow(3)$ of Theorem 1. $(3) \Rightarrow(1)$ : We have already shown this in the proof of the implication $(3) \Rightarrow(1)$ of Theorem 1 . 


\section{REFERENCES}

[1] Chevet, S., Chobanjan, S. A., Linde, W. And Tarieladze, V.I., Caractérisation de certaines classes d'espaces de Banach par les mesures gaussiennes, C. R. Acad. Sci. Paris Ser. A 285 (1977), 793-796.

[2] Chevet, S., Compacité dans l'espace des probabilités de Radon gaussiennes sur un Banach, C. R. Acad. Sci. Paris Ser. 1296 (1983), 275-278.

[3] Chevet, S., Gaussian measures and large deviations, Lecture Notes in Math. 990 (1983), 30-46.

[4] Diestel, J. ANd Uhl, J. J. Jr., Vector Measures, AMS Surveys, Providence, R. I. (1977).

[5] Fernique, X., Intégrabilité des vecteurs gaussiens, C.R. Acad. Sci. Paris Ser. A 270 (1970), 1698-1699.

[6] Grothendieck, A., Produits tensoriels topologiques et espaces nucléaires, Mem. Amer. Math. Soc. 16 (1955), 1-191.

[7] ITÔ, K. AND Nisıo, M., On the convergence of sums of independent Banach space valued random variables, Osaka J. Math. 5 (1968), 35-48.

[8] KawaBe, J., Characterization of Hilbert spaces by the strong law of large numbers (submitted to J. Multivar. Anal.).

[9] KWAPIEN, S., Isomorphic characterizations of inner product spaces by orthogonal series with vector valued coefficients, Studia Math. 44 (1972), 583-595.

[10] Linde, V., Tarieladze, V. I. And Chobanyan, S. A., Characterization of certain classes of Banach spaces by properties of Gaussian measures, Theory Prob. Appl. 25 (1980), 159-164.

[11] Perlman, M. D., Characterizing measurability, distribution and weak convergence of random variables in a Banach space by total subsets of linear functionals, J. Multivar. Anal. 2 (1972), 174-188.

[12] Prokhorov, Yu. V., Convergence of random processes and limit theorems in probability theory, Theory Prob. Appl. 1 (1956), 157-214.

[13] Umegaki, H., Weak compactness in an operator space, Kōdai Math. Sem. Rep. 8 (1956), 145-151.

[14] Vakhania, N., Probability distributions in linear spaces, Metzniereba, Tbilisi (1971).

Department of Information Sciences

Tokyo Institute of TeChNology

Oh-okayama, Meguro-ku, Tokyo, Japan 